\section{- OPEN ACCESS}

\title{
Prospective development and validation of a model to predict heart failure hospitalisation
}

\author{
R M Cubbon, ${ }^{1}$ A Woolston, ${ }^{2}$ B Adams, ${ }^{1}$ C P Gale, ${ }^{1,2}$ M S Gilthorpe, ${ }^{2}$ P D Baxter, ${ }^{2}$ \\ L C Kearney, ${ }^{1}$ B Mercer, ${ }^{1}$ A Rajwani, ${ }^{1}$ P D Batin, ${ }^{3}$ M Kahn, ${ }^{1}$ R J Sapsford, ${ }^{4}$ \\ K K Witte, ${ }^{1}$ M T Kearney ${ }^{1}$
}

${ }^{1}$ Leeds Multidisciplinary Cardiovascular Research Centre, University of Leeds, Leeds, UK

${ }^{2}$ Centre of Epidemiology and Biostatistics, University of Leeds, Leeds, UK

${ }^{3}$ Mid Yorkshire Hospitals NHS Trust, Wakefield, UK

${ }^{4}$ Leeds Teaching Hospitals NHS Trust, Leeds, UK

\section{Correspondence to} Professor Mark T Kearney, Multidisciplinary Cardiovascular Research Centre, The LIGHT Laboratories, Clarendon Way, Leeds LS2 9JT, UK; m.t.kearney@leeds.ac.uk

Received 21 November 2013 Revised 13 January 2014 Accepted 22 February 2014 Published Online First 19 March 2014
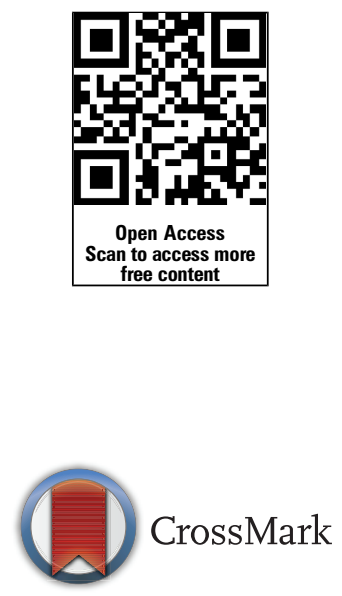

To cite: Cubbon RM, Woolston A, Adams B, et al. Heart 2014;100:923-929.

\begin{abstract}
Objective Acute heart failure syndrome (AHFS) is a major cause of hospitalisation and imparts a substantial burden on patients and healthcare systems. Tools to define risk of AHFS hospitalisation are lacking.

Methods A prospective cohort study $(n=628)$ of patients with stable chronic heart failure (CHF) secondary to left ventricular systolic dysfunction was used to derive an AHFS prediction model which was then assessed in a prospectively recruited validation cohort $(n=462)$.

Results Within the derivation cohort, 44 (7\%) patients were hospitalised as a result of AHFS during 1 year of follow-up. Predictors of AHFS hospitalisation included furosemide equivalent dose, the presence of type 2 diabetes mellitus, AHFS hospitalisation within the previous year and pulmonary congestion on chest radiograph, all assessed at baseline. A multivariable model containing these four variables exhibited good calibration (Hosmer-Lemeshow $p=0.38$ ) and discrimination (C-statistic $0.77 ; 95 \% \mathrm{Cl} 0.71$ to 0.84 ). Using a 2.5\% risk cut-off for predicted AHFS, the model defined $38.5 \%$ of patients as low risk, with negative predictive value of $99.1 \%$; this low risk cohort exhibited $<1 \%$ excess all-cause mortality per annum when compared with contemporaneous actuarial data. Within the validation cohort, an identically applied model derived comparable performance parameters (C-statistic $0.81(95 \% \mathrm{Cl} 0.74$ to 0.87$)$, Hosmer-Lemeshow $p=0.15$, negative predictive value $100 \%$ ).

Conclusions A prospectively derived and validated model using simply obtained clinical data can identify patients with CHF at low risk of hospitalisation due to AHFS in the year following assessment. This may guide the design of future strategies allocating resources to the management of CHF.
\end{abstract}

\section{INTRODUCTION}

In the USA, over 5 million individuals suffer from chronic heart failure (CHF) with direct and indirect costs of more than $\$ 30$ billion per annum. ${ }^{1}$ The main contributor to this financial burden is the cost incurred by hospitalisation of CHF patients due to acute heart failure syndrome (AHFS). In 2006, over a million hospitalisations for AHFS occurred in the USA, ${ }^{1}$ and although recent data suggest a $30 \%$ reduction in heart failure hospitalisation rates during the past decade, ${ }^{2}$ these continue to incur major economic and personal costs. ${ }^{1}$ After AHFS admission, rehospitalisation is high, ${ }^{3}$ and in some series AHFS has been shown to be a strong residual predictor of increased risk of death at 1 year, ${ }^{4}$ supporting the possibility that the natural history of CHF may be altered unfavourably by episodes of AHFS. ${ }^{5}{ }^{6}$ A large number of studies have been performed with the aim of developing models that identify patients with $\mathrm{CHF}$ at high risk of mortality. ${ }^{78}$ Despite the ongoing importance of hospitalisation due to AHFS, few studies have attempted to develop models that can specifically stratify risk of AHFS hospitalisation. ${ }^{9}$ The small number of studies that have produced models did so with the aim of predicting heart failure related rehospitalisation, as opposed to studying unselected CHF populations, and did not offer robust performance (C-statistic $\approx 0.6) .{ }^{10-12}$ These studies highlight the difficulty in deriving hospitalisation risk prediction models for use in individual heart failure patients. ${ }^{9}$ The present prospective multi-centre cohort study was specifically designed to identify and validate simple clinical variables that would stratify risk of adverse outcomes in stable outpatients with CHF.

\section{METHODS}

This was a prospective multi-centre cohort study designed to validate previous models predicting death and mode of death in patients with CHF, in addition to identifying markers of increased risk of AHFS hospitalisation in stable outpatients with CHF secondary to left ventricular systolic dysfunction. Patients $(n=628)$ included in the derivation cohort were recruited between July 2006 and January 2009. A second validation cohort $(n=462)$ was recruited between February 2009 and December 2011. All patients were recruited in specialist physician-led cardiology outpatient clinics in National Health Service tertiary or district hospitals in West Yorkshire, UK, and provided written informed consent. The study was approved by appropriate local ethics committees and conducted according to the principles expressed in the Declaration of Helsinki.

\section{Entry criteria}

Adult patients ( $>18$ years) were eligible to take part in the study if they had stable symptoms and signs of CHF (no change in New York Heart Association (NYHA) class or diuretic regimen during past 3 months) with echocardiographic evidence of left ventricular systolic dysfunction (LV EF $\leq 45 \%)$. 


\section{Baseline data collection}

At the time of recruitment into the study, a case record form detailing baseline clinical and demographic data was completed for all patients. The presence of type 2 diabetes was defined at study recruitment on the basis of detailed past medical and drug history. An erect posteroanterior chest radiograph was performed for each patient and reported by a radiologist blinded to patient characteristics (as previously described). ${ }^{13}$ A venous blood sample was taken at rest for assessment of electrolyte concentration, urea, creatine, liver function and plasma glucose. Estimated glomerular filtration rate (eGFR) was calculated using the Modification of Diet in Renal Disease method. ${ }^{14}$ A 2D echocardiogram was performed and reported by local cardiac sonographers, blinded to patient characteristics, according to British Society of Echocardiography recommendations. ${ }^{15}$ Left ventricular dimensions and EFs were calculated according to recommended guidelines. ${ }^{16}$ NYHA class was allocated according to standard criteria. ${ }^{17}$ Furosemide equivalent dose was calculated in patients prescribed bumetanide assuming that $1 \mathrm{mg}$ bumetanide was equivalent to $40 \mathrm{mg}$ furosemide. Ramipril and bisoprolol equivalent doses were derived according to our previously published work. ${ }^{18}$

The 24-hour ambulatory ECGs (Lifecard CF, Spacelabs Healthcare, Washington, USA) were obtained during normal, unrestricted, out of hospital activity as previously reported. ${ }^{19}$ Recordings were analysed with a Spacelabs Sentinel system by independent technical staff blinded to patient characteristics. ${ }^{13}$

\section{Assessment of hospitalisation and mortality}

AHFS hospitalisation was a priori defined as a new onset or worsening of signs and symptoms of heart failure with evidence of fluid overload requiring at least $24 \mathrm{~h}$ overnight hospitalisation and the use of intravenous diuretics. ${ }^{20}$ This was assessed with the use of institutional clinical event databases detailing all admissions in recruiting centres. All patients were registered with the UK Office of Population Censuses and Surveys to provide details of death, including location and date. Mortality was assessed as a binary event, censored after the first year of follow-up.

\section{Statistics}

Statistical analyses were performed using SPSS V.16.0 (SPSS Inc., Chicago, Illinois, USA) and R (V.2.8.1). Descriptive group data are presented as mean $(95 \% \mathrm{CI})$ and percentage $(95 \% \mathrm{CI})$ for continuous and categorical data, respectively. Statistical significance at the 5\% level was adopted unless stated otherwise. Univariable predictors of AHFS were derived using binary logistic regression analysis. Assessment for non-linearity of risk of association and the correct means of accounting for this were defined using a generalised additive model (see Results section). A binary logistic multivariable model was derived by initially entering all variables in figure 1 and subsequently removing those accounting for less residual variance than the degrees of freedom, or not reaching statistical significance at the $10 \%$ level using the Wald test; these data were used in categorical format where specific units are not given in figure 1, and no partitioning of continuous data was performed. Model discrimination was defined using receiver operating characteristic curves, and calibration was assessed using the Hosmer-Lemeshow statistic. The threshold of predicted AHFS risk used to denote low risk in the derivation analysis was defined on the basis of achieving negative predictive value of $>99 \%$ in order to minimise false negative labelling. A bootstrap analysis was used to assess the internal validity of the model. Five hundred random samples of the cohort were used to define the $95 \%$ centiles for model performance parameters; these intervals did not alter when fewer samples were assessed, and so analysis was not performed beyond 500 samples.

For validation of the statistical model produced from the derivation cohort, identical variables and weightings (displayed in table 2) were then applied to our prospectively recruited validation cohort. Model discrimination and calibration were

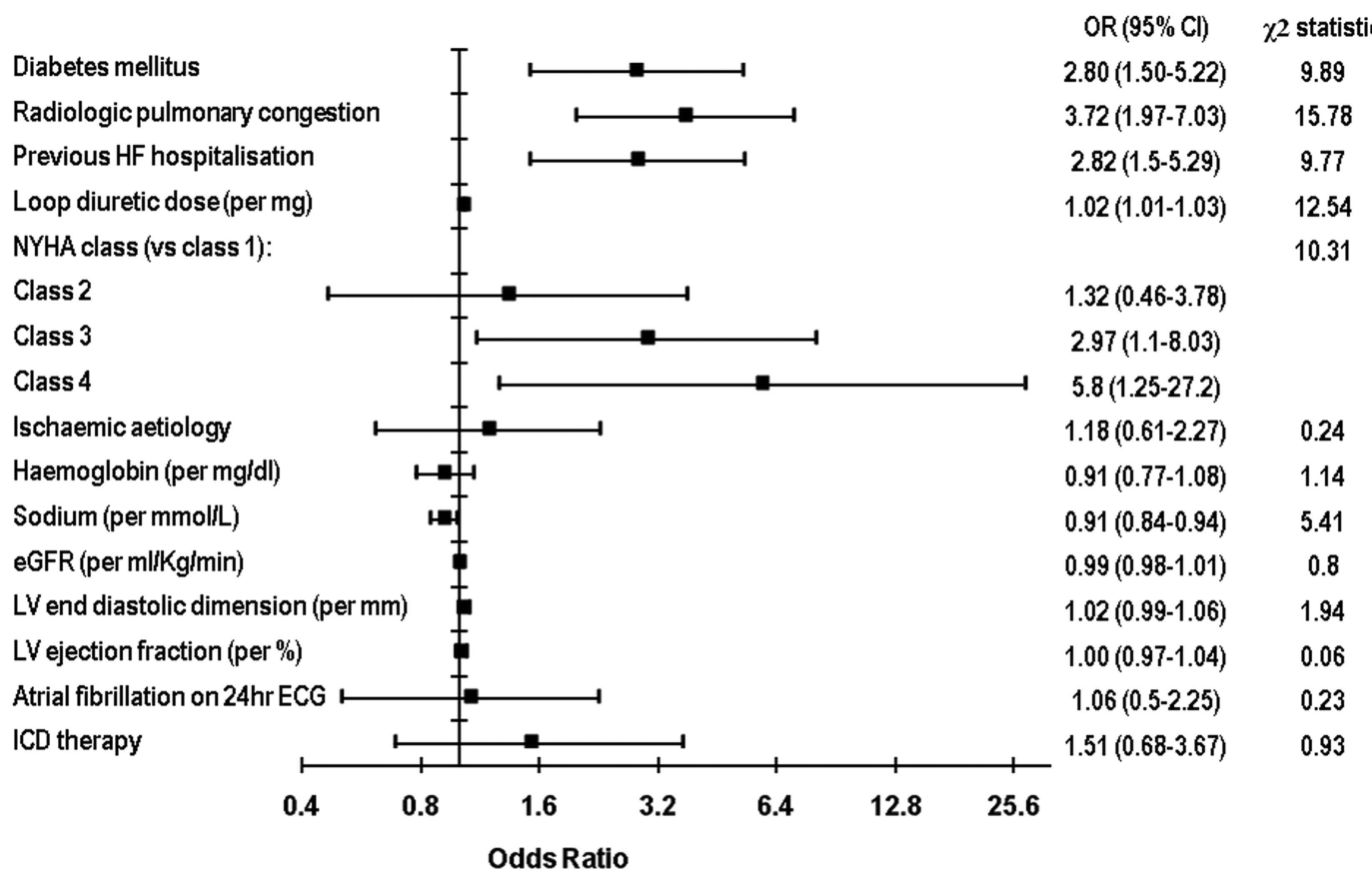

Figure 1 Univariable predictors of AHFS. Forest plot demonstrating association of selecting variables with risk of AHFS. The accompanying OR and $95 \% \mathrm{Cl}$ are presented, along with $\chi^{2}$ statistics, for each variable. AHFS, acute heart failure syndrome; eGFR, estimated glomerular filtration rate; HF, heart failure; ICD, implantable cardioverter defibrillator; NYHA, New York Heart Association. 
Table 1 Characteristics of patients with and without AHFS admission

\begin{tabular}{|c|c|c|c|}
\hline & $\begin{array}{l}\text { All }(95 \% \mathrm{Cl}) \\
\mathrm{n}=628\end{array}$ & $\begin{array}{l}\text { No AHFS }(95 \% \mathrm{Cl}) \\
\mathrm{n}=584\end{array}$ & $\begin{array}{l}\text { AHFS }(95 \% \mathrm{Cl}) \\
\mathrm{n}=44\end{array}$ \\
\hline Age (years) & 67 (66 to 68$)$ & 67.1 (66.1 to 68.2$)$ & $65.3(62.1$ to 68.5$)$ \\
\hline Male gender (\%) & 73.7 (70.1 to 77.1$)$ & 73.1 (69.3 to 76.6$)$ & $81.8(66.8$ to 91.3$)$ \\
\hline Ischaemic aetiology (\%) & $64.8(60.9$ to 68.5$)$ & $64.4(60.5$ to 68.4$)$ & $68.2(52.3$ to 80.9$)$ \\
\hline Diabetes (\%) & 24.5 (21.2 to 28.1$)$ & 22.9 (19.6 to 26.6$)$ & $45.5(30.7$ to 61.0$)$ \\
\hline HF hospitalisation in past year (\%) & 22.8 (19.6 to 26.3$)$ & $21.2(18.0$ to 24.8$)$ & $43.2(28.7$ to 58.9$)$ \\
\hline NYHA class & 2.19 (2.13 to 2.25$)$ & 2.16 (2.1 to 2.23$)$ & 2.55 (2.31 to 2.78 ) \\
\hline Systolic BP (mm Hg) & 121.9 (120.1 to 123.6$)$ & 122 (120.1 to 123.8$)$ & $120.2(112.9$ to 127.5$)$ \\
\hline Diastolic BP (mm Hg) & 71.7 (70.7 to 72.7$)$ & 71.8 (70.7 to 72.8$)$ & 71.6 (67 to 76.1 ) \\
\hline $\mathrm{BMI}\left(\mathrm{Kg} / \mathrm{m}^{2}\right)$ & 28.2 (27.6 to 28.7$)$ & 28 (27.5 to 28.6$)$ & 30.2 (27.8 to 32.5$)$ \\
\hline Heart rate (bpm) & 73.2 (71.7 to 74.7$)$ & 72.8 (71.3 to 74.3$)$ & 78.5 (71.9 to 85.1$)$ \\
\hline $\mathrm{Hb}(\mathrm{g} / \mathrm{dL})$ & $13.8(13.6$ to 13.9$)$ & 13.8 (13.6 to 13.9$)$ & 13.5 (12.8 to 14.1$)$ \\
\hline Sodium (mmol/L) & 139.3 (139 to 139.5$)$ & 139.4 (139.1 to 139.6) & 138 (136.7 to 139.3$)$ \\
\hline eGFR (mL/Kg/min) & 53.3 (52 to 54.6$)$ & $53.4(52.1$ to 54.8$)$ & $51.9(46.6$ to 57.3$)$ \\
\hline LV end diastolic diameter $(\mathrm{mm})$ & 58.7 (57.9 to 59.4$)$ & $58.5(57.7$ to 59.3$)$ & 60.7 (57.9 to 63.5$)$ \\
\hline LV end systolic diameter (mm) & 49.1 (48.3 to 49.9$)$ & 48.9 (48.0 to 49.8$)$ & $51.4(48.2$ to 54.6$)$ \\
\hline LV EF $(\%)$ & 31.3 (30.6 to 32$)$ & 31.3 (30.5 to 32$)$ & $31.6(28.7$ to 34.6$)$ \\
\hline Radiological pulmonary congestion (\%) & 24.9 (21.5 to 28.6$)$ & 22.8 (19.5 to 26.6$)$ & 52.4 (36.6 to 67.7$)$ \\
\hline Furosemide dose at baseline (mg) & 55.7 (51.5 to 59.8$)$ & 53.3 (49.1 to 57.6$)$ & 86.4 (70.9 to 101.9$)$ \\
\hline Ramipril dose at baseline (mg) & $5.0(4.7$ to 5.3$)$ & 5.1 (4.8 to 5.4$)$ & $3.5(2.7$ to 4.3$)$ \\
\hline Bisoprolol dose at baseline $(\mathrm{mg})$ & $3.2(3.0$ to 3.5$)$ & 3.3 (3.1 to 3.5$)$ & 2.9 (2.0 to 3.8$)$ \\
\hline
\end{tabular}

AHFS, acute heart failure syndrome; BMI, Body Mass Index; BP, blood pressure; eGFR, estimated glomerular filtration rate; HF, heart failure; NYHA, New York Heart Association.

assessed as outlined above, as were bootstrap analyses. An identical predicted risk of AHFS $(<2.5 \%)$ was also applied as the cut-off to define low risk group when assessing sensitivity, specificity, negative predictive value and positive predictive value.

\section{RESULTS}

Of the 738 patients screened, 628 fulfilled the entrance criteria (those excluded were predominantly because of LV EF $>45 \%$ on baseline echocardiogram). Patient characteristics are shown in table 1. Mean age was 67 (95\% CI 66 to 68 ) years; $73.7 \%$ were men; $20.9 \%$ were categorised as NYHA functional class I, $41.7 \%$ as class II, $34.8 \%$ as class III and $2.6 \%$ as class IV. Mean eGFR was 53.3 (52 to 54.6$) \mathrm{mL} / \mathrm{Kg} / \mathrm{min}$ and mean sodium was 139.3 (139 to 139.5 ) $\mathrm{mmol} / \mathrm{L}$. Ischaemic heart disease was the primary aetiology of $\mathrm{CHF}$ in $64.8 \%$, and $24.5 \%$ of patients were diagnosed with type 2 diabetes mellitus. Mean LV EF was 31.3 (30.6 to 32) \% and left ventricular end diastolic diameter was 58.7 (57.9 to 59.4) mm. ACE inhibitors or angiotensin receptor blockers were prescribed to $88 \%$, $\beta$-adrenoceptor blockers ( $\beta$-blockers) to $79.2 \%$ and mineralocorticoid receptor antagonists to $41 \% ; 23.7 \%$ received CRT-P/D within 6 months of recruitment. The mean dose of furosemide prescribed was 55.7 (51.5 to 59.8$) \mathrm{mg}$.

One year hospitalisation rate was $7 \%$ for AHFS episodes $(n=44), 14 \%$ for all cardiovascular related episodes (including AHFS) and $20.7 \%$ for all non-elective (including cardiovascular) episodes occurring during follow-up. In patients with AHFS, the median (IQR) time from recruitment to first AHFS was 165 (94 to 267) days. One year all-cause mortality rate was $9.2 \%$ (58 patients; 32 inhospital deaths).

\section{Predictors of AHFS}

An initial analysis of univariable predictors of AHFS based upon clinically plausible variables is outlined in figure 1. AHFS hospitalisation within the previous year, the presence of type 2 diabetes mellitus, pulmonary congestion on chest radiograph, furosemide dose, NYHA class and serum sodium concentration were all statistically significant predictors of AHFS. Notably, assessment for non-linearity of risk of association using a generalised additive model revealed that a quadratic correction term should be applied to furosemide dose; this was applied in all univariable and multivariable analyses. A multivariable regression model containing the former four factors (table 2) was produced after initially entering all factors in figure 1 and removing those accounting for less residual variance than the degrees of freedom or not reaching statistical significance. This exhibited excellent calibration (Hosmer-Lemeshow $\mathrm{p}=0.38$ ) and discrimination (C-statistic 0.77 (95\% CI 0.71 to 0.84); figure 2A). Furthermore, this model offered only low discrimination in predicting non-AHFS related hospitalisation (C-statistic $0.61(0.54$ to 0.68 ); figure $2 \mathrm{~B}$ ).

Using a cut-off value of $<2.5 \%$ predicted AHFS to denote low risk, sensitivity was $95.2 \%$, specificity $41 \%$, negative predictive value $99.1 \%$ and positive predictive value $10.9 \% ; 38.5 \%$

Table 2 Multivariable model for prediction of AHFS hospitalisation

\begin{tabular}{lc}
\hline & OR $(95 \% \mathrm{Cl})$ \\
\hline Diabetes mellitus & $1.933(0.971$ to 3.848$)$ \\
Radiological pulmonary congestion & $2.641(1.358$ to 5.136$)$ \\
Previous HF hospitalisation & $2.136(1.077$ to 4.237$)$ \\
Loop diuretic dose (per mg) & $1.025(1.004$ to 1.046$)$ \\
Loop diuretic dose quadratic term & $0.9999(0.9998$ to 1.0000$)$ \\
Prevalence of AHFS hospitalisation for reference & $1.1(0.4$ to 2.8$) \%$ \\
population* & \\
\hline
\end{tabular}

* Reference population: no diabetes, no pulmonary congestion, no previous HF hospitalisation, loop diuretic dose $=0$.

AHFS, acute heart failure syndrome; HF, heart failure. 
Table 3 Model performance metrics according to risk threshold applied

\begin{tabular}{llccrc}
\hline Predicted risk threshold (\%) & Sensitivity (\%) & Specificity (\%) & PPV (\%) & NPV (\%) & Proportion 'high' risk (\%) \\
\hline 2.5 & $95.2(85.7$ to 100$)$ & $41(15.6$ to 58.8$)$ & $10.9(74$ to 13.3$)$ & $99.1(97.7$ to 100$)$ & 61.5 \\
5 & $76.2(65.7$ to 93.2$)$ & $58.8(43.9$ to 74.3$)$ & $12.2(10.1$ to 17.6$)$ & $97(96.3$ to 98.9$)$ & 43.7 \\
10 & $61.9(39.4$ to 74.5$)$ & $80.7(70.6$ to 88.8$)$ & $19.4(13.2$ to 27.3$)$ & $96.6(94.9$ to 97.7$)$ & 22.4 \\
15 & $38.1(18.2$ to 61.7$)$ & $90.4(83.4$ to 95$)$ & $23(15.9$ to 32.9$)$ & $95.1(94.1$ to 96.8$)$ & 11.6 \\
30 & $9.5(2.2$ to 32.9$)$ & $98.6(95.6$ to 99.7$)$ & $33.2(10$ to 64.9$)$ & $93.5(93.2$ to 95.2$)$ & 2 \\
\hline
\end{tabular}

Figures in brackets represent $95 \%$ Cls determined by Bootstrap analyses.

NPV, negative predictive value, PPV, positive predictive value.

of the population were deemed low risk. Furthermore, annual mortality in the low risk group was $3.5 \%$; when compared with UK actuarial mortality data, ${ }^{21}$ the excess CHF-related mortality was $0.7 \%$ per annum, compared with an excess of $9.9 \%$ in the higher risk group. The performance parameters of the model at other thresholds of predicted AHFS hospitalisation risk are also outlined in table 3. These data indicate that application of higher predicted risk thresholds may allow definition of groups at 'high' risk of AHFS, although this may be relevant to only a small percentage of the total population.

Bootstrap analyses for the C-statistic $(0.79$ (95th centiles 0.72 to 0.85$))$, sensitivity $(96 \%(85.7 \%$ to $100 \%))$, specificity $(38.1 \%$ $(15.6 \%$ to $58.8 \%))$, positive predictive value $(10.2 \%(7.4 \%$ to $13.3 \%)$ ) and negative predictive value $(99.2 \% \quad(97.7 \%$ to $100 \%)$ ) indicate reasonable internal validity of the model.

\section{Validation cohort}

Using identical inclusion and exclusion criteria, a further cohort of 462 patients was then recruited to validate the performance of the model in predicting low risk of AHFS; baseline characteristics are presented in table 4 . One year hospitalisation rate was $4.5 \%$ for AHFS episodes $(n=21), 11.3 \%$ for all cardiovascular related episodes and $30.3 \%$ for all non-elective episodes. In patients with AHFS, the median (IQR) time from recruitment to first AHFS was 64 (41 to 136) days. One year all-cause mortality rate was $7.1 \%$ (33 patients; 17 inhospital deaths).

Using the variables and their respective weightings identified in the derivation cohort (table 2), individual risk of AHFS was predicted. Using these parameters, the model continued to show satisfactory calibration (Hosmer-Lemeshow $\mathrm{p}=0.15)$ and similar discrimination (C-statistic 0.81 (95\% CI 0.74 to 0.87 ) by bootstrap analysis; figure 3). Applying an identical threshold of $<2.5 \%$ predicted risk of AHFS to define low risk, $29.4 \%$ of the population were deemed low risk, sensitivity was $100 \%$ (95\% CI $100 \%$ to $100 \%)$, specificity was $30.8 \%$ (95\% CI $26.3 \%$ to $34.5 \%)$, negative predictive value was $100 \%(100 \%$ to $100 \%)$ and positive predictive value was $6.6 \%(6.2 \%$ to $6.9 \%)$. One year all-cause mortality in the low risk group was $4.5 \%(n=6)$, which is $1.2 \%$ in excess of actuarial data, ${ }^{21}$ while in the higher risk group, mortality was $8.4 \%(n=27)$, which is $4.4 \%$ in excess of actuarial data.

\section{DISCUSSION}

The principal findings of the present report are that three simple questions ((1) Does the patient have diabetes? (2) Did the patient have an AHFS hospitalisation in the past year? (3) What daily dose of loop diuretic is the patient requiring?) and a chest radiograph can provide information that identifies patients at low risk of subsequent AHFS hospitalisation. These measurements have the potential to allow individually tailored, patient-centred therapeutic approaches, targeting of sophisticated monitoring systems or differing levels of intensity of treatment/monitoring.

\section{Identifying patients at increased risk of hospitalisation due to AHFS}

The goal of early detection of AHFS is to decrease hospitalisation and mortality, prevent worsening $\mathrm{CHF}$ and improve the quality of life of patients with CHF, and their families. Our data
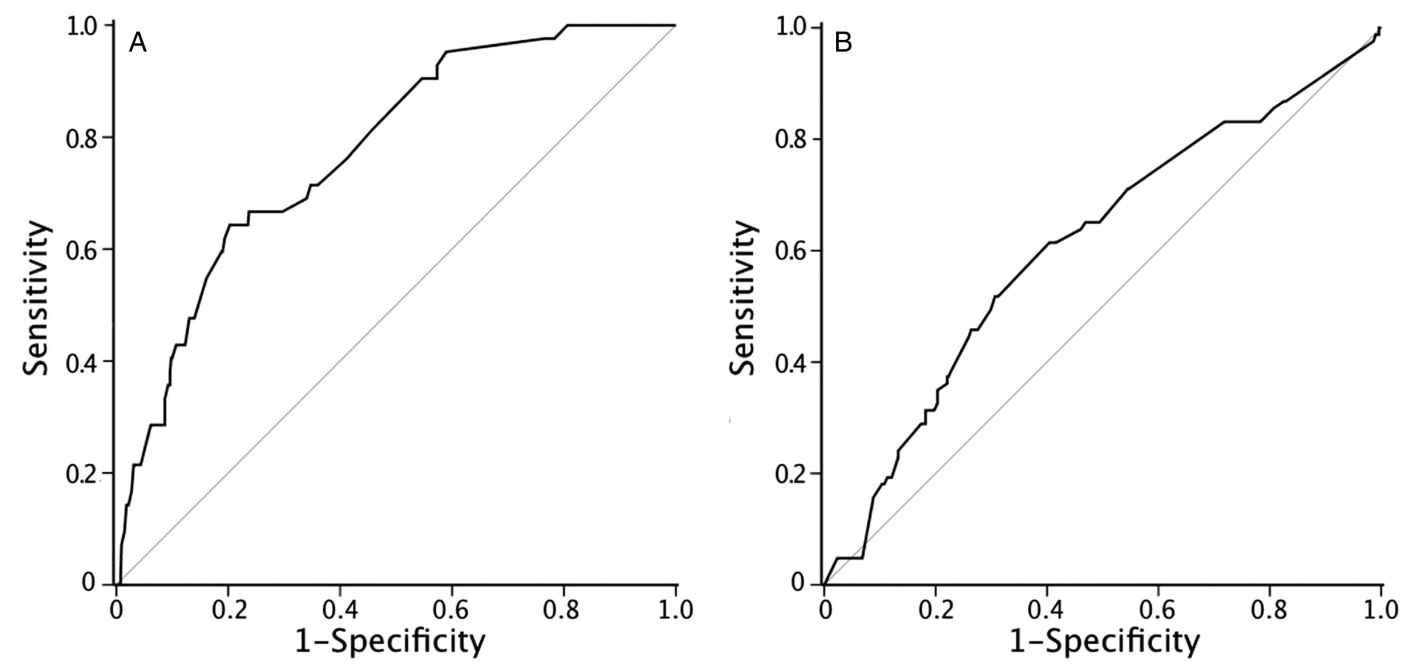

Figure 2 Model discrimination in derivation cohort. Receiver operating characteristic curves demonstrating the discriminative capacity of the multivariable model detailed in table 3 to predict (A) AHFS hospitalisation and (B) non-AHFS hospitalisation. AHFS, acute heart failure syndrome. 
Table 4 Characteristics of the validation cohort

\begin{tabular}{lc}
\hline & $(95 \% \mathrm{Cl}) \mathrm{n}=462$ \\
\hline Age (years) & $69.9(68.9 \text { to } 71.0)^{*}$ \\
Male gender (\%) & $73.3(69.3$ to 77.4$)$ \\
Ischaemic aetiology (\%) & $61.0(56.6$ to 65.5$)$ \\
Diabetes (\%) & $28.4(24.2$ to 32.5$)$ \\
HF hospitalisation in past year (\%) & $15.4(12.1 \text { to } 18.7)^{*}$ \\
NYHA class & $2.07(2.0 \text { to } 2.13)^{*}$ \\
Systolic BP (mm Hg) & $119.8(117.9$ to 121.8$)$ \\
Diastolic BP (mm Hg) & $71.6(70.5$ to 72.6$)$ \\
BMI (Kg/m²) & $27.0(25.3$ to 28.7$)$ \\
Heart rate (bpm) & $76.4(74.1$ to 78.7$)$ \\
Hb (g/dL) & $14.0(12.6 \text { to } 15.4)^{*}$ \\
Sodium (mmol/L) & $139.2(138.9$ to 139.5$)$ \\
eGFR (mL/Kg/min) & $55.9(53.6 \text { to } 58.2)^{*}$ \\
LV end diastolic diameter (mm) & $57.8(57.0$ to 58.5$)$ \\
LV EF (\%) & $33.1(32.2 \text { to } 33.9)^{*}$ \\
Radiological pulmonary congestion (\%) & $8.6(6.0 \text { to } 11.2)^{*}$ \\
Furosemide dose at baseline (mg) & $48.9(44.6 \text { to } 53.3)^{*}$ \\
\hline
\end{tabular}

*Denotes $p<0.05$ in comparison with derivation cohort.

BMI, Body Mass Index; BP, blood pressure; eGFR, estimated glomerular filtration rate; HF, Heart Failure; NYHA, New York Heart Association.

demonstrate that, by integrating information from a clinical history (loop diuretic dose, previous AHFS hospitalisation and the presence of type 2 diabetes mellitus) with chest radiograph data, it is possible to identify patients with CHF at very low risk (below 1\% per annum) of AHFS hospitalisation and a subgroup at relatively high risk (which can be further stratified by the model). These data may allow more appropriate use of limited healthcare resources. Furthermore, patients defined as having low risk of AHFS by our model also appear to be at low additional risk of all-cause death when compared with relevant actuarial data (approximately 1\% excess risk).

\section{AHFS hospitalisation: pathophysiology and predictors}

AHFS, defined as a gradual or rapid change in heart failure signs and symptoms resulting in a need for urgent therapy,

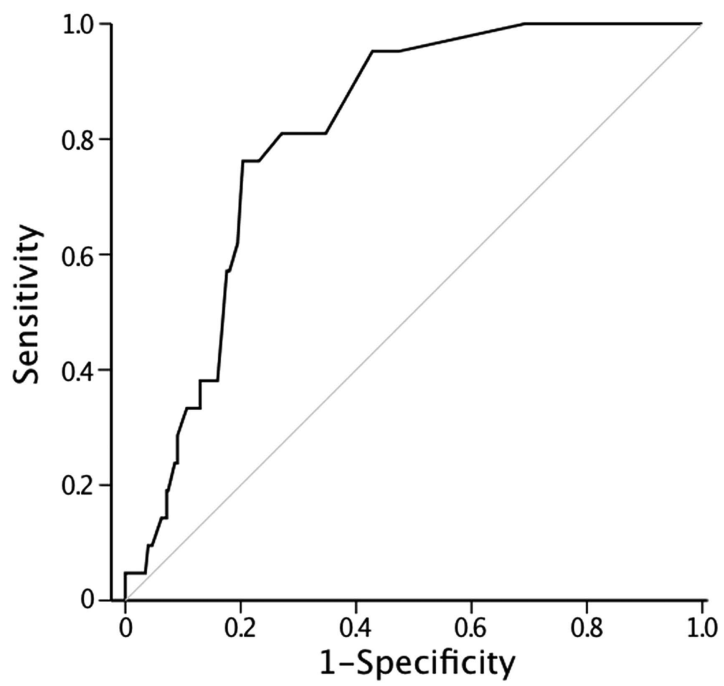

Figure 3 Model discrimination in validation cohort. Receiver operating characteristic curve demonstrating the discriminative capacity of the identically applied multivariable model detailed in table 3 to predict acute heart failure syndrome hospitalisation. poses a unique challenge to clinicians caring for patients with $\mathrm{CHF}^{22}$ The majority of patients hospitalised with AHFS have worsening CHF associated with previously documented left ventricular systolic impairment. ${ }^{23}$ In community patients with $\mathrm{CHF}$, mortality significantly increases after each hospitalisation ${ }^{24}$ and risk of death from progressive heart failure soon after hospital discharge is increased substantially. ${ }^{4}$ Therefore, AHFS hospitalisation in patients with CHF may be a marker of CHF progression and/or may increase mortality due to the impact of therapies used in hospital to rapidly reverse decompensation.

A recent systematic review demonstrated the paucity of data identifying specific and accurate predictors of AHFS hospitalisation. ${ }^{9}$ Though there have been a number of studies evaluating predictors of readmission after an AHFS, they have been limited to patients with a prior AHFS admission and have had relatively short periods of follow-up. Moreover, no studies to date have elected to examine prediction of AHFS hospitalisation a priori in highly characterised patients in a number of centres. As a result, and despite the size of the problem, prognostic models specifically aimed at AHFS are currently lacking; therefore, our ability to target therapeutic strategies aimed at preventing AHFS in selected groups of patients is lacking.

\section{Pulmonary congestion and AHFS}

The symptoms and signs of AHFS are thought to be principally due to severe pulmonary congestion secondary to elevated left ventricular filling pressure. ${ }^{20}$ Cardiopulmonary congestion is thought to be the principal reason for AHFS admissions and readmissions; ${ }^{20}$ this may start weeks before presentation with AHFS. $^{25} 26$ Cardiopulmonary congestion leads to additional neurohumoral activation and subendocardial ischaemia. ${ }^{27} 28 \mathrm{In}$ keeping with myocyte necrosis, available data suggest an increase in serum troponin in patients with AHFS. ${ }^{29}$ Moreover, progressive mitral and/or tricuspid regurgitation due to altered cardiac size and shape may occur, leading to an accelerated decline in left ventricular systolic performance. ${ }^{30}$ In keeping with these previous studies, we have demonstrated that evidence of pulmonary congestion on a plain chest radiograph (indirect evidence of increased left ventricular filling pressure) is associated with an increased risk of AHFS hospitalisation.

\section{Functional capacity and AHFS}

The NYHA class is a 4-point semiquantitative index of a patient's functional ability. ${ }^{17}$ In previous studies it has been shown to correlate with quality of life, ${ }^{31}$ quantitative assessment of cardiopulmonary performance such as peak $\mathrm{VO}_{2}{ }^{32}$ and prognosis. ${ }^{33}$ In the present report, we have demonstrated that NYHA class is a univariable predictor of AHFS hospitalisation. This is not surprising in view of the fact that NYHA appears to be a good indicator of more advanced heart failure. However, the reproducibility of NYHA class has been questioned, ${ }^{34}$ and this may underlie the loss of NYHA class during the development of our multivariable model. It is likely that loop diuretic dose is a surrogate of symptomatic status which, by virtue of its objective measurement and continuous distribution, offers greater predictive value than NYHA class, thereby resulting in its inclusion in the final model. To the authors' knowledge, no data have previously been published demonstrating the residual value of loop diuretic dose in predicting AHFS hospitalisation risk after accommodating other major risk factors.

\section{Previous heart failure hospitalisation and AHFS}

As discussed, in patients with CHF, mortality significantly increases after each hospitalisation and risk of death from 
progressive heart failure soon after hospital discharge is increased substantially. ${ }^{4} 24$ Therefore, AHFS hospitalisation in patients with CHF appears to be a marker of CHF progression and/or may increase mortality due to the impact of aggressive therapies used in hospital to reverse decompensation. ${ }^{35} 36$ In keeping with this, our dataset shows that previous hospitalisation for AHFS is a residual and powerful predictor of further AHFS hospitalisation.

\section{Type 2 diabetes mellitus and AHFS}

In the present study, approximately $25 \%$ of patients were classified as suffering from type 2 diabetes. In a large study from Scotland, MacDonald et $a l^{37}$ showed that diabetes was associated with an increased risk of readmission to hospital in patients admitted to hospital with a principal diagnosis of heart failure irrespective of EF. Data from the CHARM study showed that hospitalisation in patients with $\mathrm{CHF}$ and diabetes, with both preserved or reduced EFs, was higher. ${ }^{38}$ Our dataset is consistent with these studies, demonstrating in ambulant outpatients that the presence of diabetes increases the risk of heart failure hospitalisation.

\section{UTILITY OF THE MODEL}

For a model to be adopted in clinical practice it must answer simply a clearly defined question, thereby providing the clinician and patient with a high degree of confidence in the anticipated outcome. Our decision to apply a low predicted risk threshold for the application of the model clearly defines this as a means to predict low risk of AHFS hospitalisation, and the negative predictive value $(99.1 \%$ and $100 \%$ in derivation and validation cohorts) means that $<1 \%$ of 'low risk' patients will experience AHFS hospitalisation. Clearly, the lower the threshold chosen, the greater the negative predictive value will become, though we feel that our application of the model achieves an acceptable balance between achieving a low false negative rate, while deeming a large group of patients as low risk. Indeed, since approximately a third of the population are deemed low risk, major reallocation of finite resources, perhaps through novel care strategies, can be contemplated. For example, low risk patients may be able to receive lower intensity monitoring, hence allowing available specialist resources to be directed at reducing hospitalisation in higher risk patients; such strategies of course require prospective validation. Reassurance that such a strategy would be appropriate comes from our mortality data, indicating an approximate $1 \%$ excess all-cause mortality (compared with actuarial data) in the low risk groups of derivation and validation cohorts. Moreover, the broad repetition of all of our findings in a prospectively recruited validation cohort suggests applicability in routine clinical practice. Finally, it is notable that the use of higher predicted risk thresholds can allow our model identify groups at higher risk of AHFS (see table 4), although this is evidently relevant to a much smaller proportion of the cohort.

\section{Study limitations}

The present dataset presents a number of markers of increased risk of AHFS hospitalisation in patients with CHF due to left ventricular systolic dysfunction. While the model developed has good internal calibration and discrimination, which was confirmed locally in a prospectively recruited validation cohort, any model should be validated and calibrated in different populations and locations to ensure wider transportability and generalisability. The study design aimed to assess routine clinical measurements, and so we did not measure more novel markers of risk, such as brain natriuretic peptide (BNP) or markers of systemic inflammation; ${ }^{39} 40$ these may add further prognostic information to the present model. In addition, the present study did not assess patients with CHF and preserved EF, and so the model cannot be applied to this group of patients. Next, while all patients attending recruiting clinics were approached for consent to participation, it is impossible to exclude any selection bias in our derivation and validation cohorts. The comparable results in both cohorts make selection bias seem less likely, although reproduction of our findings in geographically distinct cohorts would add further support to our findings. Finally, it should be noted that patient compliance with medical therapy may be an important factor in precipitating AHFS- unfortunately, we have no data pertaining to this in either cohort.

\section{CONCLUSIONS}

We have shown that information obtained from clinical history and a plain chest radiograph can identify patients with $\mathrm{CHF}$ at low risk of subsequent hospitalisation for AHFS. This information may be useful in tailoring therapeutic strategies for patients with $\mathrm{CHF}$.

\section{Key messages}

What is already known on this subject

Hospitalisation due to acute heart failure syndromes imparts a substantial burden on individuals and healthcare systems. Currently, there is no way of predicting the risk of these events in individual patients.

\section{What this study adds}

We have derived a model able to predict very low risk of heart failure related hospitalisation in patients with stable chronic heart failure associated with left ventricular systolic dysfunction during the subsequent year. This model was then validated in a second prospectively recruited cohort, and demonstrated comparable performance metrics.

Contributors All authors have contributed to study design, data collection or analysis; drafting of the manuscript; final approval to submitting manuscript.

Funding The study was funded by the British Heart Foundation (PG/08/020/24617).

Ethics approval Leeds Central Research Ethics Committee.

Provenance and peer review Not commissioned; externally peer reviewed.

Open Access This is an Open Access article distributed in accordance with the Creative Commons Attribution Non Commercial (CC BY-NC 3.0) license, which permits others to distribute, remix, adapt, build upon this work non-commercially, and license their derivative works on different terms, provided the original work is properly cited and the use is non-commercial. See: http://creativecommons.org/ licenses/by-nc/3.0/

\section{REFERENCES}

1 Rosamond W, Flegal K, Furie K, et al. Heart disease and stroke statistics-2008 update: a report from the American Heart Association Statistics Committee and Stroke Statistics Subcommittee. Circulation 2008;117:e25-146.

2 Chen J, Normand S, Wang Y, et al. National and regional trends in heart failure hospitalization and mortality rates for medicare beneficiaries, 1998-2008. JAMA 2011;306:1669-78.

3 Ross JS, Chen J, Lin Z, et al. Recent national trends in readmission rates after heart failure hospitalization. Circ Heart Fail 2010;3:97-103.

4 Solomon SD, Dobson J, Pocock S, et al. Influence of nonfatal hospitalization for heart failure on subsequent mortality in patients with chronic heart failure. Circulation 2007;116:1482-7. 
5 Lee DS, Austin PC, Stukel TA, et al. "Dose-dependent" impact of recurrent cardiac events on mortality in patients with heart failure. Am J Med 2009;122:162.

6 Setoguchi S, Stevenson LW, Schneeweiss S. Repeated hospitalizations predict mortality in the community population with heart failure. Am Heart $J$ 2007; 154:260-6.

7 Lee DS, Austin PC, Rouleau JL, et al. Predicting mortality among patients hospitalized for heart failure: derivation and validation of a clinical model. JAMA 2003;290:2581-7.

8 Mozaffarian D, Anker SD, Anand I, et al. Prediction of mode of death in heart failure: the seattle heart failure model. Circulation 2007:116:392-8.

9 Ross JS, Mulvey GK, Stauffer B, et al. Statistical models and patient predictors of readmission for heart failure: a systematic review. Arch Intern Med 2008;168:1371-86.

10 Keenan PS, Normand SL, Lin Z, et al. An administrative claims measure suitable for profiling hospital performance on the basis of 30-day all-cause readmission rates among patients with heart failure. Circ Cardiovasc Qual Outcomes 2008;1:29-37.

11 Philbin E, DiSalvo T. Prediction of hospital readmission for heart failure: development of a simple risk score based on administrative data. J Am Coll Cardiol 1999:33:1560-6.

12 Yamokoski L, Hasselblad V, Moser D, et al. Prediction of rehospitalization and death in severe heart failure by physicians and nurses of the ESCAPE trial. J Card Fail 2007;13:8-13

13 Kearney MT, Fox KAA, Lee AJ, et al. Predicting death due to progressive heart failure in patients with mild-to-moderate chronic heart failure. J Am Coll Cardiol 2002:40:1801-8.

14 Levey AS, Bosch JP, Lewis JB, et al. A more accurate method to estimate glomerular filtration rate from serum creatinine: a new prediction equation. Ann Int Med 1999;130:461-70.

15 British Society of Echocardiography. http://www.bsecho.org (accessed 20 Mar 2010).

16 Lang RM, Bierig M, Devereux RB, et al. Recommendations for chamber quantification: a report from the American Society of Echocardiography's guidelines and standards committee and the chamber quantification writing group, developed in conjunction with the European Association of Echocardiography, a Branch of the European Society of Cardiology. J Am Soc Echocardiog 2005;18:1440-63.

17 The Criteria Committee of the New York Heart Association. Nomenclature and criteria for diagnosis of diseases of the heart and great vessels. 9th edn. Boston, Mass: Little, Brown \& Co., 1994

18 Cubbon RM, Gale CP, Kearney LC, et al. Changing characteristics and mode of death associated with chronic heart failure caused by left ventricular systolic dysfunction: a study across therapeutic eras. Circ Heart Fail 2011;4:396-403.

19 Nolan J, Batin PD, Andrews R, et al. Prospective study of heart rate variability and mortality in chronic heart dailure: results of the United Kingdom Heart Failure Evaluation and Assessment of Risk Trial (UK-Heart). Circulation 1998:98:1510-16.

20 Gheorghiade M, Zannad F, Sopko G, et al. Acute heart failure syndromes: current state and framework for future research. Circulation 2005;112:3958-68.

21 United Kingdom National Statistics Publications Hub. http://www.statistics.gov.uk/ STATBASE/ssdataset.asp?vlnk=9552E (accessed 18 Feb 2010)

22 Gheorghiade M, Pang PS. Acute heart failure syndromes. J Am Coll Cardiol 2009:53:557-73
23 Adams J, Fonarow GC, Emerman CL, et al. Characteristics and outcomes of patients hospitalized for heart failure in the United States: rationale, design, and preliminary observations from the first 100,000 cases in the Acute Decompensated Heart Failure National Registry (ADHERE). Am Heart J 2005;149:209-16.

24 Dunlay SM, Redfield MM, Weston SA, et al. Hospitalizations after heart failure diagnosis: a community perspective. J Am Coll Cardiol 2009;54:1695-702.

25 Androne AS, Katz SD, Lund $L$, et al. Hemodilution is common in patients with advanced heart failure. Circulation 2003:107:226-9.

26 Chaudhry SI, Wang Y, Concato J, et al. Patterns of weight change preceding hospitalization for heart failure. Circulation 2007:116:1549-54.

27 Beohar N, Erdogan AK, Lee DC, et al. Acute heart failure syndromes and coronary perfusion. J Am Coll Cardiol 2008;52:13-16.

28 Milo 0 , Cotter G, Kaluski E, et al. Comparison of inflammatory and neurohormonal activation in cardiogenic pulmonary edema secondary to ischemic versus nonischemic causes. Am J Cardiol 2003;92:222-6.

29 Peacock WF IV, De Marco T, Fonarow GC, et al. Cardiac troponin and outcome in acute heart failure. N Engl J Med 2008;358:2117-26.

30 Kono T, Sabbah HN, Rosman H, et al. Left ventricular shape is the primary determinant of functional mitral regurgitation in heart failure. J Am Coll Cardiol 1992;20:1594-8.

31 Green $\mathrm{CP}$, Porter CB, Bresnahan DR, et al. Development and evaluation of the Kansas City Cardiomyopathy Questionnaire: a new health status measure for heart failure. J Am Coll Cardiol 2000;35:1245-55.

32 Russell SD, Saval MA, Robbins JL, et al. New York Heart Association functional class predicts exercise parameters in the current era. Am Heart J 2009:158:S24-30.

33 Levy WC, Mozaffarian D, Linker DT, et al. The Seattle Heart Failure Model: prediction of survival in heart failure. Circulation 2006;113:1424-33.

34 Kubo SH, Schulman S, Starling RC, et al. Development and validation of a patient questionnaire to determine New York heart association classification. J Card Fail 2004:10:228-35.

35 Francis $G$, Sigel R, Goldsmith $S$, et al. Acute vasoconstrictor response to intravenous furosemide in patients with chronic congestive heart failure. Ann Int Med 1985;103:1-6.

36 Silver MA, Horton DP, Ghali JK, et al. Effect of nesiritide versus dobutamine on short-term outcomes in the treatment of patients with acutely decompensated heart failure. J Am Coll Cardiol 2002;39:798-803.

37 MacDonald MR, Jhund PS, Petrie MC, et al. Discordant short- and long-term outcomes associated with diabetes in patients with heart failure: importance of age and sex: a population study of 5.1 million people in Scotland. Circ Heart Fail 2008; 1:234-41.

38 MacDonald MR, Petrie MC, Varyani F, et al. Impact of diabetes on outcomes in patients with low and preserved ejection fraction heart failure. Eur Heart $J$ 2008:29:1377-85.

39 Logeart D, Thabut G, Jourdain P, et al. Predischarge B-type natriuretic peptide assay for identifying patients at high risk of re-admission after decompensated heart failure. J Am Coll Cardiol 2004;43:635-41.

40 Radauceanu A, Ducki C, Virion JM, et al. Extracellular matrix turnover and inflammatory markers independently predict functional status and outcome in chronic heart failure. J Card Fail 2008;14:467-74 